\title{
Méthodes de crible et fonctions sommes des chiffres
}

\author{
par \\ E. Fouvry (Orsay) et C. Mauduit (Marseille)
}

I. Introduction. Soit $\left(c_{n}\right)$ une suite de nombres complexes, $\mathcal{Q}$ et $\mathcal{R}$ deux applications, qui à tout entier $q \geq 1$ associent deux ensembles $\mathcal{Q}(q)$ et $\mathcal{R}(q)$ de classes de congruences modulo $q$, vérifiant $\mathcal{Q}(q) \subset \mathcal{R}(q)$.

Par définition, on dira que le réel $\vartheta$ est un exposant de répartition (au sens fort) pour la suite $\left(c_{n}\right)$, relativement aux fonctions $\mathcal{Q}$ et $\mathcal{R}$, si, pour tout $\varepsilon>0$ et tout $A>0$, on a, pour $x \geq 1$, l'égalité

$$
\begin{aligned}
\sum_{q<x^{\vartheta}-\varepsilon} \max _{y \leq x} \max _{a \in \mathcal{Q}(q)} \mid \sum_{\substack{n<y \\
n \equiv a(\bmod q)}} c_{n} & -\frac{1}{|\mathcal{R}(q)|} \sum_{\substack{n<\mathcal{N} \\
n \in \mathcal{R}(q)}} c_{n} \mid \\
& =O_{\varepsilon, A}\left(\left(\sum_{n<x}\left|c_{n}\right|\right)(\log 2 x)^{-A}\right),
\end{aligned}
$$

avec les conventions que, si $\mathcal{Q}(q)$ est vide, le terme correspondant est nul et que $|\mathcal{E}|$ désigne le cardinal de l'ensemble $\mathcal{E}$.

L'égalité (1.1) est une façon d'apprécier l'harmonie de la répartition en moyenne de $\left(c_{n}\right)$ dans les progressions arithmétiques. Le choix de la fonction $\mathcal{R}$ s'impose de lui-même pour mener à des situations intéressantes, les situations les plus connues étant $\mathcal{R}(q)=\mathbb{Z} / q \mathbb{Z}$ et $\mathcal{R}(q)=(\mathbb{Z} / q \mathbb{Z})^{*}$, le terme correspondant dans (1.1) apparaît alors comme un terme principal dont l'étude est en général aisée.

Des expressions semblables à (1.1) apparaissent de façon cruciale dans des problèmes de crible, la suite $\left(c_{n}\right)$ étant alors positive et les estimations obtenues étant très sensibles à la valeur de $\vartheta$. L'estimation (1.1) n'est en fait nécessaire que pour un choix très particulier de $\mathcal{Q}$, à savoir $\mathcal{Q}=\mathcal{Q}_{b}$, où $b$ est un entier fixé et la fonction $\mathcal{Q}_{b}$ est définie par $\mathcal{Q}_{b}(q)=\{b\}$ ou $\emptyset$, suivant que $(b, q)=1$ ou $(b, q)>1$. Enfin, signalons que si la suite $\left(c_{n}\right)$ n'est pas trop anarchique et assez dense, il est possible, par un argument de découpage, d'insérer le terme $\max _{y \leq x}$ dans une expression (1.1) dont l'exactitude n'est connue que dans le cas particulier $y=x$ (voir [Gra], Lemma 6 et [Fo-T], paragraphe 3.1, par exemple). 
Rappelons quelques cas de suites $\left(c_{n}\right)$ où on sait que $\vartheta=1$ est un exposant de répartition :

- $c_{n}=1$ avec $\mathcal{Q}(q)=\mathcal{R}(q)=\mathbb{Z} / q \mathbb{Z}$,

- $c_{n}=\mu^{2}(n)$, fonction caractéristique des nombres sans facteur carré, avec $\mathcal{Q}=\mathcal{Q}_{b}$ et $\mathcal{R}(q)=(\mathbb{Z} / q \mathbb{Z})^{*}$, le symbole $O$ de (1.1) dépendant alors de l'entier $b$ choisi,

- $c_{n}$ nombre de représentations de l'entier $n$ comme somme de deux nombres premiers avec, par exemple, $\mathcal{Q}(q)=\{1\}$ et $\mathcal{R}(q)=(\mathbb{Z} / q \mathbb{Z})^{*}$, ceci est une conséquence de l'inégalité de Cauchy-Schwarz et du Théorème de Barban-Davenport-Halberstam ([Iw], p. 213, par exemple).

A cette liste, il convient de rajouter le cas surprenant où $c_{n}$ est le nombre de diviseurs de $n$ : en effet, (1.1) est vrai pour $\mathcal{Q}=\mathcal{Q}_{b}$ et $\mathcal{R}(q)=(\mathbb{Z} / q \mathbb{Z})^{*}$, à condition d'exclure, dans la sommation sur $q$, l'intervalle $\left[x^{2 / 3-\varepsilon}, x^{2 / 3+\varepsilon}\right]$ ([Fo], Corollaire 5). Si on adopte une définition plus générale de l'exposant de répartition, on sait maintenant que la suite $\left(c_{n}\right)$ nombre de représentations de l'entier $n$ sous la forme $m^{2}+a^{2}$ (avec $m$ entier quelconque et $a \in \mathcal{A}$ où $\mathcal{A}$ est une suite quelconque mais assez dense) a pour exposant de répartition $1([\mathrm{Fo}-\mathrm{I}])$.

Il faut aussi rappeler que pour $c_{n}$ fonction caractéristique des nombres premiers, la plus grande valeur de $\vartheta$ pour laquelle on sache que (1.1) est vrai pour $\mathcal{Q}(q)=\mathcal{R}(q)=(\mathbb{Z} / q \mathbb{Z})^{*}$ est $\vartheta=1 / 2$ (théorème de BombieriVinogradov) et qu'on pense que (1.1) est vrai pour $\vartheta=1$ (conjecture d'Elliott-Halberstam).

L'objet de cet article est de donner des exemples de suites à exposant de répartition très proche de 1 .

Soit $r \geq 2$ un entier, $s_{r}(n)$ la somme des chiffres dans le développement en base $r$ de l'entier $n$. Nous nous intéressons à la répartition dans les progressions arithmétiques de la suite des entiers $n$, tels que $s_{r}(n)$ appartienne à une classe de congruence imposée.

Notre résultat principal est le

ThÉORÈme. Soient $r \geq 2, b$ et $d$ des entiers tels que $(d, r-1)=1$. Alors, pour $x \geq 1$, pour tout $A$ et tout $\varepsilon>0$, on a l'égalité

$$
\begin{array}{r}
\sum_{q<x^{\vartheta_{r}-\varepsilon}} \max _{y<x} \max _{1 \leq a \leq q}\left|\sum_{\substack{n<y, s_{r}(n) \equiv b(\bmod d) \\
n \equiv a(\bmod q)}} 1-\frac{1}{q} \sum_{n<y, s_{r}(n) \equiv b(\bmod d)} 1\right| \\
=O\left(x(\log 2 x)^{-A}\right),
\end{array}
$$

pour $\vartheta_{r}=1-\log M(r) / \log r$. Dans cet énoncé, la constante $O$ dépend au plus de $d, r, A$ et $\varepsilon$ et la fonction $M(r)$ est définie par 


$$
M(r)=\frac{1}{n} \sum_{k=0}^{n-1} \frac{1}{\cos \frac{2 k+1}{4 n} \pi}
$$

ou

$$
M(r)=\frac{1}{2 n+1}\left(1+2 \sum_{k=1}^{n} \frac{1}{\cos \frac{k \pi}{2 n+1}}\right),
$$

suivant que $r$ est pair $(r=2 n)$ ou $r$ impair $(r=2 n+1)$.

Une étude précise de la constante $M(r)$ conduira, via le crible pondéré de Greaves, au

Corollaire 1. Soient $d$ et $r \geq 2$ deux entiers tels que $(d, r-1)=1$. Alors, pour tout entier $b$, on a pour $x \rightarrow \infty$ la minoration

$$
\mid\left\{n \leq x: s_{r}(n) \equiv b(\bmod d), n=p_{1} \text { ou } n=p_{1} p_{2}\right\} \mid \gg \frac{x}{\log x} .
$$

Nous verrons au paragraphe VI (voir (6.3)) que le point important de ce théorème est le fait que $\vartheta_{r}$ tende vers 1 lorsque $r \rightarrow \infty$. Le corollaire suivant est une application directe du crible de Bombieri, dans la version très agréable qu'en ont donnée Friedlander et Iwaniec [Fr-I2]. Rappelons que la fonction généralisée de von Mangoldt d'ordre $k(k$ entier $\geq 1)$ est $\Lambda_{k}=(\log )^{k} * \mu$, et qu'elle a pour support l'ensemble des entiers ayant au plus $k$ facteurs premiers. On prouve le

Corollaire 2. Soient $b, d, k$ et $r$ des entiers vérifiant $k \geq 2, r \geq 2$ et $(d, r-1)=1$. Il existe alors $x_{0}=x_{0}(d, k, r)$ tel que pour $x \geq x_{0}$ on a l'égalité

$$
\sum_{n<x, s_{r}(n) \equiv b(\bmod d)} \Lambda_{k}(n)=\frac{k}{d} x(\log x)^{k-1}\left(1+O\left(\frac{(\log \log r)^{5}}{\log r}\right)\right),
$$

où la constante $O$ dépend au plus de $d$ et $k$.

Ce corollaire montre donc que plus la base $r$ est grande, meilleur est l'encadrement de la somme étudiée.

II. Transformation du problème. Nous passons aux sommes trigonométriques en écrivant la fonction caractéristique des entiers $n$ tels que $n \equiv a$ $(\bmod q)$ et $s_{r}(n) \equiv b(\bmod d)$ sous la forme

$$
\frac{1}{d q} \sum_{\xi^{q}=1} \sum_{\zeta^{d}=1} \xi^{n-a} \zeta^{s_{r}(n)-b}
$$

Soit $R_{r}(y ; a, q ; b, d)$ le terme apparaissant dans (1.2) à l'intérieur du symbole $|\cdot|$. Il devient 


$$
R_{r}(y ; a, q ; b, d)=\frac{1}{d q} \sum_{\substack{\xi^{q}=1 \\ \xi \neq 1}} \sum_{\zeta^{d}=1} \sum_{n<y} \xi^{n-a} \zeta^{s_{r}(n)-b} .
$$

En écartant le terme $\zeta=1$ dont la contribution est en $O(1 / d)$, on a l'égalité

$$
R_{r}(y ; a, q ; b, d)=\frac{1}{d q} \sum_{\substack{\xi^{q}=1 \\ \xi \neq 1}} \sum_{\substack{\zeta^{d}=1 \\ \zeta \neq 1}} T_{r}(y ; a, \xi ; b, \zeta)+O(1 / d),
$$

avec

$$
T_{r}(y ; a, \xi ; b, \zeta)=\sum_{n<y} \xi^{n-a} \zeta^{s_{r}(n)-b} .
$$

On peut supposer que $y$ est un entier $\leq x$ que l'on décompose en base $r$ sous la forme

$$
y=\sum_{i=0}^{s} \varepsilon_{i}(y) r^{i}
$$

avec $\varepsilon_{s}(y) \geq 1$. Posons $y_{s}=\varepsilon_{s}(y) r^{s}$. En décomposant l'intervalle de sommation sur $n$ on a l'égalité

$$
T_{r}(y ; a, \xi ; b, \zeta)=T_{r}\left(y_{s} ; a, \xi ; b, \zeta\right)+\xi^{y_{s}} \zeta^{\varepsilon_{s}(y)} T_{r}\left(y-y_{s} ; a, \xi ; b, \zeta\right) .
$$

Dans (2.2), l'entier $y-y_{s}$ vérifie maintenant $\varepsilon_{s}\left(y-y_{s}\right)=0$, donc cette formule se prête fort bien à une itération. En prenant les modules, l'égalité (2.1) devient

$$
\left|R_{r}(y ; a, q ; b, d)\right| \leq \frac{1}{d q} \sum_{u \in \mathcal{X}} \sum_{\substack{\xi^{q}=1 \\ \xi \neq 1}} \sum_{\substack{\zeta^{d}=1 \\ \zeta \neq 1}}\left|T_{r}^{*}(u ; \xi, \zeta)\right|+O(1 / d),
$$

où $\mathcal{X}$ désigne l'ensemble des entiers inférieurs à $x$ de la forme $k r^{j}$ avec $1 \leq k<r$ et $j \geq 0$ et où on a défini

$$
T_{r}^{*}(u ; \xi, \zeta)=\sum_{0 \leq n<u} \xi^{n} \zeta^{s_{r}(n)}
$$

L'existence et l'unicité du développement en base $r$ de tout entier $n$ fournit l'égalité de factorisation

$$
T_{r}^{*}\left(k r^{N} ; \xi, \zeta\right)=\left(\sum_{l=0}^{k-1} \zeta^{l} \xi^{l r^{N}}\right) \prod_{0 \leq n<N}\left(\sum_{l=0}^{r-1} \zeta^{l} \xi^{l r^{n}}\right),
$$

pour $N$ entier quelconque et $1 \leq k<r$. Dans l'expression (2.4), le premier facteur est plus petit que $r$ en module. Puisque le nombre d'éléments de $\mathcal{X}$ est $O_{r}(\log x)$, en regroupant (2.3) et (2.4), on voit que la partie gauche de 
(1.2) est en

$$
\begin{array}{r}
O\left(\log x \cdot \max _{\substack{\zeta^{d}=1 \\
\zeta \neq 1}} \max _{N \leq(\log x) /(\log r)} \sum_{q<x^{\vartheta_{r}-\varepsilon}} q^{-1} \sum_{\substack{\xi^{q}=1 \\
\xi \neq 1}}\left|\prod_{0 \leq n<N}\left(\sum_{l=0}^{r-1} \zeta^{l} \xi^{l r^{n}}\right)\right|\right) \\
+O\left(x^{\vartheta_{r}-\varepsilon}\right) .
\end{array}
$$

En posant maintenant $\zeta=\exp (2 \pi i j / d)$ et $\xi=\exp (2 \pi i k / q)$ on a l'identité

$$
\left|\sum_{l=0}^{r-1} \zeta^{l} \xi^{l r^{n}}\right|=\frac{\left|\sin \pi\left(\frac{j}{d} r+\frac{k}{q} r^{n+1}\right)\right|}{\left|\sin \pi\left(\frac{j}{d}+\frac{k}{q} r^{n}\right)\right|}=\left|\varphi\left(r^{n} \frac{k}{q}, \frac{j}{d}\right)\right|,
$$

avec

$$
\varphi(\alpha, \beta)=\frac{\sin \pi r(\alpha+\beta)}{\sin \pi(\alpha+\beta)},
$$

si $\alpha+\beta$ est non entier, et $\varphi(\alpha, \beta)=(-1)^{(\alpha+\beta)(r-1)} r$, dans le cas contraire.

Posons

$$
F_{N}(\alpha, \beta)=\prod_{0 \leq n<N} \varphi\left(r^{n} \alpha, \beta\right) .
$$

Grâce à ces notations et à la relation (2.5), on voit que la preuve du Théorème se ramène à montrer que la relation

$$
\sum_{Q<q \leq 2 Q} \sum_{k=1}^{q-1}\left|F_{N}(k / q, j / d)\right|=O\left(Q x(\log 2 x)^{-A}\right)
$$

est exacte pour tout $Q \leq x^{\vartheta_{r}-\varepsilon}$, pour tout $0<j<d$, pour tout $N \leq$ $(\log x) /(\log r)$ et pour tout $A$.

III. Étude de $\left\|F_{N}\right\|_{\infty}$. Nous aurons besoin du lemme suivant lors de la preuve du Théorème :

Lemme 1. Soient $r$ et $d$ des entiers tels que $(d, r-1)=1$. Alors, il existe une constante absolue $M(r, d)<r$ telle que pour tout $N \geq 0$ et tout entier $0<j<d$, on ait la relation

$$
\left\|F_{N}(\cdot, j / d)\right\|_{\infty}=O_{r}\left(M(r, d)^{N}\right) .
$$

Signalons que cette relation est triviale pour $M(r, d)=r$ (puisque $|\varphi(\alpha, \beta)| \leq r)$ et qu'une étude plus élaborée conduit à des valeurs intéressantes de cette constante $M(r, d)$ et, ainsi, à des résultats sur la répartition dans une progression arithmétique fixée des entiers $n$ tels que $s_{r}(n) \equiv$ $b(\bmod d)(\operatorname{voir}[\mathrm{Ge}])$.

D'après l'expression de la fonction $F_{N}$ en produit de la fonction continue $\varphi$, on voit, en regroupant les termes deux par deux, que, pour démontrer ce 
lemme, il suffit de prouver l'inégalité

$$
|\varphi(t, j / d) \varphi(r t, j / d)|<r^{2},
$$

pour tout $t$ et tout $0<j<d$. S'il existait un $t$ tel que $|\varphi(t, j / d) \varphi(r t, j / d)|=$ $r^{2}$, en revenant à l'écriture de $|\varphi|$ sous la forme

$$
|\varphi(t, j / d)|=\left|\sum_{l=0}^{r-1} \exp (2 \pi i(l j / d+l t))\right|,
$$

on voit que $t$ vérifierait simultanément les relations $j / d+t \equiv 0(\bmod 1)$ et $j / d+r t \equiv 0(\bmod 1)$, ce qui entraînerait $j(r-1) / d \equiv 0(\bmod 1)$, ce qui est contraire à l'hypothèse du Lemme 1 qui est ainsi démontré.

IV. Étude de $\left\|F_{N}\right\|_{1}$. L'objet de ce paragraphe est de majorer l'intégrale

$$
I_{N}(r, \beta)=\int_{0}^{1}\left|F_{N}(t, \beta)\right| d t
$$

uniformément par rapport à $\beta$, pour $N$ et $r$ tendant vers l'infini. En partant de la définition (2.6), en découpant l'intervalle d'intégration en intervalles de la forme $[k / r,(k+1) / r]$ puis en faisant le changement de variable $u=r t-k$, on parvient à l'égalité

$$
I_{N}(r, \beta)=\int_{0}^{1} \psi_{1}(t, \beta)\left|F_{N-1}(t, \beta)\right| d t
$$

avec

$$
\psi_{1}(t, \beta)=\frac{1}{r} \sum_{0 \leq k<r} \varphi\left(\frac{t+k}{r}, \beta\right)=\frac{1}{r} \sum_{0 \leq k<r}\left|\frac{\sin \pi(t+r \beta)}{\sin \pi\left(\frac{t+k}{r}+\beta\right)}\right| .
$$

Ce processus peut fort bien s'itérer, faisant apparaître des fonctions $\psi_{2}$, $\psi_{3}, \ldots$ construites par récurrence à partir de $\psi_{1}$; nous n'aurons pas besoin de ce processus qui a été exploité dans [Fo-M].

Posons

$$
\Psi_{r}(u)=\frac{1}{r} \sum_{0 \leq k<r}\left|\frac{\sin \pi r u}{\sin \pi(u+k / r)}\right| .
$$

La formule (4.1) conduit clairement à l'inégalité

$$
I_{N}(r, \beta) \leq\left\|\Psi_{r}\right\|_{\infty} I_{N-1}(r, \beta) \leq\left\|\Psi_{r}\right\|_{\infty}^{N} .
$$

L'étude de $\left\|F_{N}\right\|_{1}$ se ramène ainsi à celle de $\left\|\Psi_{r}\right\|_{\infty}$. La fonction $\Psi_{r}(u)$ est de période $1 / r$, ceci nous autorise à n'étudier les valeurs de $\Psi_{r}(u)$ que pour $0 \leq u \leq 1 / r$ où cette fonction a l'expression plus agréable

$$
\Psi_{r}(u)=\frac{1}{r} \sum_{0 \leq k<r} \frac{\sin \pi r u}{\sin \pi(u+k / r)},
$$


où n'apparaissent que des termes positifs. Notre but est de rattacher la fonction $\Psi_{r}$ à la fonction $M(r)$ définie dans le Théorème sous forme du

Lemme 2. Pour tout entier $r \geq 2$, on a l'égalité

$$
\left\|\Psi_{r}\right\|_{\infty}=M(r) \text {. }
$$

Pour faire apparaître au numérateur et au dénominateur la même quantité, on écrit la formule (4.3) sous la forme

$$
\Psi_{r}(u)=\frac{1}{r} \sum_{0 \leq k<r}(-1)^{k} \frac{\sin \pi r(u+k / r)}{\sin \pi(u+k / r)},
$$

puis la démonstration se scinde en deux cas suivant la parité de $r$.

(i) Cas où $r=2 n$ est pair. On écrit l'égalité

$$
(-1)^{k} \frac{\sin \pi r(u+k / r)}{\sin \pi(u+k / r)}=2(-1)^{k} \sum_{j=0}^{n-1} \cos \left((2 j+1)\left(u+\frac{k}{2 n}\right) \pi\right),
$$

d'où l'égalité

$$
\begin{aligned}
r \Psi_{r}(u) & =2 \sum_{k=0}^{2 n-1}(-1)^{k} \sum_{j=0}^{n-1} \cos \left((2 j+1)\left(u+\frac{k}{2 n}\right) \pi\right) \\
& =2 \sum_{j=0}^{n-1} \sum_{k=0}^{2 n-1}(-1)^{k} \cos \left((2 j+1)\left(u+\frac{k}{2 n}\right) \pi\right),
\end{aligned}
$$

puis en utilisant la formule

$$
\sum_{k=0}^{m-1}(-1)^{k} \cos (a+h k)=\frac{\cos \left(a+\frac{m-1}{2} h+\frac{m-1}{2} \pi\right) \sin \left(\frac{m h}{2}+\frac{m \pi}{2}\right)}{\cos \frac{h}{2}},
$$

on parvient à l'égalité

$$
\begin{aligned}
r \Psi_{r}(u) & =2 \sum_{j=0}^{n-1} \frac{\cos \left((2 j+1) \pi u+\frac{2 n-1}{2} \cdot \frac{2 j+1}{2 n} \pi+\frac{2 n-1}{2} \pi\right) \sin \left(n \frac{(2 j+1) \pi}{2 n}+n \pi\right)}{\cos \frac{(2 j+1) \pi}{4 n}} \\
& =2 \sum_{j=0}^{n-1} \frac{\cos \left((2 j+1)\left(u-\frac{1}{4 n}\right) \pi\right)}{\cos \frac{2 j+1}{4 n} \pi} .
\end{aligned}
$$

On voit alors que pour $0 \leq u \leq 1 / r$, la fonction $\Psi_{r}(u)$ est concave, et, puisqu'elle vérifie l'égalité $\Psi_{r}(u)=\Psi_{r}(1 / r-u)$, elle atteint son maximum au point $u=1 /(4 n)$, où elle vaut $M(r)$.

(ii) Cas où $r=2 n+1$ est impair. On écrit l'égalité

$$
(-1)^{k} \frac{\sin \pi r(u+k / r)}{\sin \pi(u+k / r)}=(-1)^{k}+2(-1)^{k} \sum_{j=1}^{n} \cos \left(2 j \pi\left(u+\frac{k}{2 n+1}\right)\right) \text {, }
$$


d'où la relation

$$
\begin{aligned}
r \Psi_{r}(u) & =1+2 \sum_{j=1}^{n} \sum_{k=0}^{2 n}(-1)^{k} \cos \left(2 j \pi\left(u+\frac{k}{2 n+1}\right)\right) \\
& =1+2 \sum_{j=1}^{n} \frac{\cos \left(2 j \pi u+n \frac{2 j \pi}{2 n+1}+n \pi\right) \sin \left(\frac{2 n+1}{2} \cdot \frac{2 j \pi}{2 n+1}+\frac{2 n+1}{2} \pi\right)}{\cos \frac{j \pi}{2 n+1}} \\
& =1+2 \sum_{j=1}^{n} \frac{\cos \left(2 j \pi\left(u-\frac{1}{4 n+2}\right)\right)}{\cos \frac{j \pi}{2 n+1}} .
\end{aligned}
$$

Écrite ainsi, la fonction $\Psi_{r}(u)$ est une somme de fonctions concaves sur $[0,1 / r]$, symétrique par rapport à $u=1 /(4 n+2)$, où elle atteint donc son maximum. Ceci termine la preuve du Lemme 2.

V. Démonstration du théorème. Notre démarche est très proche de celle de $[\mathrm{Fo}-\mathrm{M}]$. Pour montrer la relation (2.7), on rend tout d'abord les fractions $k / q$ irréductibles, d'où l'égalité

$$
\begin{aligned}
\sum_{Q<q \leq 2 Q} \sum_{k=1}^{q-1}\left|F_{N}(k / q, j / d)\right| & =\sum_{\delta=1}^{2 Q} \sum_{Q \delta^{-1}<q \leq 2 Q \delta^{-1}} \sum_{\substack{k=1 \\
(k, q)=1}}^{q-1}\left|F_{N}(k / q, j / d)\right| \\
& :=\sum_{\delta=1}^{2 Q} G_{N}\left(Q \delta^{-1}, j / d\right),
\end{aligned}
$$

par définition. On introduit un paramètre $N_{1} \leq N$, qui permet de découper $F_{N}$ en

$$
F_{N}(t, j / d)=F_{N_{1}}(t, j / d) \cdot F_{N-N_{1}}\left(r^{N_{1}} t, j / d\right),
$$

ce qui, grâce au Lemme 1, donne la relation

$$
\left|F_{N}(t, j / d)\right|=O\left(M(r, d)^{N-N_{1}}\left|F_{N_{1}}(t, j / d)\right|\right),
$$

et, enfin

$$
G_{N}\left(Q \delta^{-1}, j / d\right)=O\left(M(r, d)^{N-N_{1}} G_{N_{1}}\left(Q \delta^{-1}, j / d\right)\right) .
$$

On applique l'inégalité de Sobolev-Gallagher ([Fo-M], Lemme 1), puisque les points $k / q$ sont $\delta^{2} /\left(4 Q^{2}\right)$ bien espacés, on a donc la majoration

$$
G_{N_{1}}\left(Q \delta^{-1}, j / d\right) \ll Q^{2} \delta^{-2}\left\|F_{N_{1}}(\cdot, j / d)\right\|_{1}+\left\|F_{N_{1}}^{\prime}(\cdot, j / d)\right\|_{1} .
$$

La définition (2.6) de la fonction $F_{N}$ donne, par dérivation par rapport à $t$, l'inégalité

$$
\left|F_{N_{1}}^{\prime}(t, \xi)\right| \ll_{r} \sum_{0 \leq i<N_{1}} r^{i} \prod_{\substack{j<N_{1} \\ j \neq i}}\left|\varphi\left(r^{j} t, \xi\right)\right|
$$


d'où la relation

$$
\left\|F_{N_{1}}^{\prime}(\cdot, j / d)\right\|_{1}=O_{r}\left(\sum_{i<N_{1}} r^{i} \cdot r^{N_{1}-i}\left\|F_{i}(\cdot, j / d)\right\|_{1}\right)=O\left(r^{N_{1}}\left\|\Psi_{r}\right\|_{\infty}^{N_{1}}\right)
$$

d'après (4.2). En regroupant (5.3) et (5.4), on a

$$
G_{N_{1}}\left(Q \delta^{-1}, j / d\right) \ll_{r}\left\|\Psi_{r}\right\|_{\infty}^{N_{1}}\left(Q^{2} \delta^{-2}+r^{N_{1}}\right),
$$

ce qui nous incite à prendre pour $N_{1}$ le minimum de la partie entière de $2 \log (Q / \delta) / \log r$ et de $N$. La relation (5.2) devient alors

$$
\begin{aligned}
& G_{N}\left(Q \delta^{-1}, j / d\right) \\
& \quad=O\left(M(r, d)^{N}\left(\frac{r\left\|\Psi_{r}\right\|_{\infty}}{M(r, d)}\right)^{2 \log (Q / \delta) / \log r}+Q^{2} \delta^{-2}\left\|\Psi_{r}\right\|_{\infty}^{N}\right) .
\end{aligned}
$$

D'après (5.1), il reste à sommer (5.5) sur les $\delta<2 Q$. Pour faciliter la majoration de cette série, on peut supposer que $M(r, d)$, dont l'existence est assurée par le Lemme 1, est très proche de $r$, de telle façon qu'on ait l'inégalité $r\left\|\Psi_{r}\right\|_{\infty} / M(r, d) \leq \sqrt{r}$ (ceci est possible pour $r>2$ puisque $\left\|\Psi_{r}\right\|_{\infty}=M(r)$ et qu'on verra en (6.5) l'inégalité $\vartheta_{r}>1 / 2$ qui équivaut à $\left\|\Psi_{r}\right\|_{\infty}<\sqrt{r}$ ).

Avec une telle supposition, (5.5) est affaiblie en

$$
G_{N}\left(Q \delta^{-1}, j / d\right)=O\left(M(r, d)^{N} Q \delta^{-1}+Q^{2} \delta^{-2}\left\|\Psi_{r}\right\|_{\infty}^{N}\right)
$$

d'où, en sommant sur $\delta$, on a

$$
\sum_{Q<q \leq 2 Q} \sum_{k=1}^{q-1}\left|F_{N}(k / q, j / d)\right| \ll M(r, d)^{N} Q \log x+Q^{2}\left\|\Psi_{r}\right\|_{\infty}^{N} .
$$

Il suffit d'utiliser les Lemmes 1 et 2 pour constater que la relation (2.7) est démontrée pour le choix de $Q$ fixé par le théorème, dont la preuve est ainsi complète.

VI. Preuve du Corollaire 1. Dans un premier temps, il faut rendre plus parlante la valeur de l'exposant de répartition $\vartheta_{r}$ du Théorème. Nous utiliserons le fait que pour $a=\pi /(2 n)$ ou $a=\pi /(2 n+1)$, la fonction $x \mapsto 1 / \sin (x+1 / 2) a$ est positive et décroissante sur l'intervalle [0, $n-1]$.

(i) Cas où $r$ est pair $(r=2 n, r \geq 6)$. Dans ce cas, on écrit

$$
\begin{aligned}
M(r) & =\frac{1}{n} \sum_{k=0}^{n-1} \frac{1}{\sin \frac{2 k+1}{4 n} \pi}=\frac{1}{n \sin \frac{\pi}{4 n}}+\frac{1}{n \sin \frac{3 \pi}{4 n}}+\frac{1}{n \sin \frac{5 \pi}{4 n}}+\frac{1}{n} \sum_{k=3}^{n-1} \frac{1}{\sin \frac{2 k+1}{4 n} \pi} \\
& \leq \frac{1}{n \sin \frac{\pi}{4 n}}+\frac{1}{n \sin \frac{3 \pi}{4 n}}+\frac{1}{n \sin \frac{5 \pi}{4 n}}+\frac{1}{n} \int_{2}^{n-1} \frac{d x}{\sin \left(x+\frac{1}{2}\right) \frac{\pi}{2 n}} \\
& \leq \frac{1}{3 \sin \frac{\pi}{12}}+\frac{1}{3 \sin \frac{\pi}{4}}+\frac{1}{3 \sin \frac{5 \pi}{12}}+\frac{2}{\pi} \log \frac{\tan \left(\frac{\pi}{4}-\frac{\pi}{8 n}\right)}{\tan \frac{5 \pi}{8 n}},
\end{aligned}
$$


car la fonction $x / \sin x$ est croissante sur $[0, \pi / 2]$, d'où la majoration

$$
M(r) \leq \frac{1}{3 \sin \frac{\pi}{12}}+\frac{1}{3 \sin \frac{\pi}{4}}+\frac{1}{3 \sin \frac{5 \pi}{12}}-\frac{2}{\pi} \log \left(\tan \frac{5 \pi}{4 r}\right)
$$

qu'on écrit de façon plus explicite comme

$$
M(r) \leq 2.105-\frac{2}{\pi} \log \left(\tan \frac{5 \pi}{4 r}\right) .
$$

(ii) Cas où $r$ est impair $(r=2 n+1, r \geq 7)$. La méthode est fort semblable :

$$
\begin{aligned}
M(r) & =\frac{1}{2 n+1}\left(1+2 \sum_{k=0}^{r-1} \frac{1}{\sin \frac{2 k+1}{2(2 n+1)} \pi}\right) \\
& \leq \frac{1}{r}+\frac{2}{r \sin \frac{\pi}{2 r}}+\frac{2}{r \sin \frac{3 \pi}{2 r}}+\frac{2}{r \sin \frac{5 \pi}{2 r}}+\frac{2}{r} \int_{2}^{n-1} \frac{d x}{\sin \left(x+\frac{1}{2}\right) \frac{\pi}{r}} \\
& \leq \frac{1}{7}+\frac{2}{7 \sin \frac{\pi}{14}}+\frac{2}{7 \sin \frac{3 \pi}{14}}+\frac{2}{7 \sin \frac{5 \pi}{14}}-\frac{2}{\pi} \log \left(\tan \frac{5 \pi}{4 r}\right),
\end{aligned}
$$

qui s'écrit finalement

$$
M(r) \leq 2.203-\frac{2}{\pi} \log \left(\tan \frac{5 \pi}{4 r}\right) .
$$

Les relations (6.1) et (6.2) entraînent

$$
M(r)=O(\log r) \quad(r \rightarrow \infty),
$$

ce qui, par la définition de $\vartheta_{r}$ donnée lors de l'énoncé du Théorème, implique que, pour $r \rightarrow \infty$, on a

$$
\vartheta_{r}=1-O\left(\frac{\log \log r}{\log r}\right) .
$$

Pour prouver le Corollaire 1, il suffit de vérifier, grâce au crible pondéré ([H-R], Theorem 9.3) que $\vartheta_{r}$ vérifie, pour tout $r \geq 2$, une inégalité de la forme

$$
\vartheta_{r} \Lambda_{2}>1
$$

mais, grâce aux travaux de Greaves, on sait qu'on peut prendre une valeur de $\Lambda_{2}$ très proche de la valeur conjecturale $\Lambda_{2}=2$, à savoir $\Lambda_{2}=1.936$ ([Gre]), ce qui ramène la vérification de (6.4) à celle de

$$
\frac{\log M(r)}{\log r}<0.483
$$

En minorant $\vartheta_{r}$ par (6.1) ou (6.2) suivant les cas, on voit que (6.5) est vérifiée pour $r \geq 6$; ceci pourra se faire en calculant la dérivée de la fonction $r^{0.483}+\frac{2}{\pi} \log \left(\tan \frac{5 \pi}{4 r}\right)$. 
Il reste donc à traiter les petites valeurs de $r$. Pour $r=2$, on a $M(2)=$ $\sqrt{2}$, d'où $\vartheta_{2}=1 / 2$; l'inégalité (6.5) n'est pas vérifiée, mais, d'après [Fo-M], on sait, par une méthode plus élaborée que le Corollaire 1 est vrai pour cette valeur de $r$. On a $M(3)=5 / 3$, donc $\vartheta_{3}=0.5350 \ldots$ et l'inégalité (6.5) est exacte. On a de même $M(4)=2 \cos (\pi / 8), M(5)=(1+2 / \cos (\pi / 5)+$ $2 / \cos (2 \pi / 5)) / 5$, d'où $\vartheta_{4}=0.5571 \ldots, \vartheta_{5}=0.5727 \ldots$ et $(6.5)$ est toujours vérifiée. Ceci termine la preuve du Corollaire 1.

VII. Le crible asymptotique de Bombieri. On doit à Bombieri ([Bo1] et aussi [Bo2]) d'avoir démontré que pour un problème de crible linéaire appliqué à une suite $\left(c_{n}\right)$ positive ou nulle, ayant 1 pour exposant de répartition, il est possible d'accéder à un équivalent asymptotique de la somme

$$
\sum_{n<x} c_{n} \Lambda_{k}(n)
$$

pour $x \rightarrow \infty$ et $k \geq 2$. Nous avons volontairement schématisé et simplifié cet important résultat de Bombieri. Ce travail a été étendu par Friedlander et Iwaniec ([Fr-I1]) par une étude délicate des uniformités par rapport aux différents paramètres. Nous utiliserons une autre très récente extension de ce travail, qui s'adapte parfaitement à notre situation puisqu'elle traite du cas où l'exposant de répartition tend vers 1 ([Fr-I2]). Afin de rendre notre travail complet, nous rappelons dans sa plus grande généralité le résultat de Friedlander et Iwaniec.

Soit

$$
a(n) \geq 0, \quad A(x)=\sum_{n \leq x} a(n)
$$

On pose aussi

$$
A_{d}(x)=\sum_{n \leq x, d \mid n} a(n), \quad A_{d}(x)=g(d) A(x)+r_{d}(x)
$$

et

$$
R(x, D)=\sum_{d<D}^{b}\left|r_{d}(x)\right|
$$

où le ${ }^{b}$ signifie que la somme est restreinte aux entiers sans facteur carré. On choisit un nombre $z$ tel que

$$
2 \leq z \leq x^{\delta}, \quad \delta \leq \min \left(\frac{1}{20}, \frac{1}{k+1}\right) .
$$

Les hypothèses sont au nombre de huit :

$$
\begin{gathered}
R(x, x / z) \leq A(x)(\log x)^{-2}, \\
A(x / z) \leq A(x)(\log x)^{-2},
\end{gathered}
$$


$\left(\mathrm{H}_{3}\right)$

$$
\sum_{p \geq z} A_{p^{2}}(x) \log p \leq \delta A(x) .
$$

Ces trois premières hypothèses concernaient le terme d'erreur, les suivantes traitent du terme principal :

$$
g \text { est multiplicative avec } 0 \leq g(p)<1 \text {. }
$$

Il existe une constante $c \geq 2$, telle que, pour tout $u \geq w \geq 2$, on ait

$$
\sum_{w \leq p<u} \frac{g(p)}{1-g(p)} \log p \leq \log \frac{c u}{w} .
$$

Il existe une fonction $f$ vérifiant

$$
\begin{gathered}
f \text { est complètement multiplicative avec } 0 \leq f(p)<1, \\
\sum_{p \geq z}|f(p)-g(p)| \leq \delta^{3}, \\
\sum_{n \leq t} f(n)=a \log t+b+O\left((\log t)^{-2}\right),
\end{gathered}
$$

pour tout $t \geq 2$ avec $a>0$ et $b$ des constantes. On a le

Lemme 3 ([Fr-I2]). Soit $k \geq 2$ et $x>x_{0}(k)$. Supposons que les hypothèses $\left(\mathrm{H}_{1}\right)-\left(\mathrm{H}_{5}\right)$ soient vraies pour $z=x^{\delta}$ vérifiant (7.1). Supposons qu'il existe $f$ proche de $g$, autrement dit telle que les hypothèses $\left(\mathrm{H}_{6}\right)-\left(\mathrm{H}_{7}\right)$ soient satisfaites. Sous ces hypothèses, on a l'égalité

$$
\sum_{n \leq x}^{b} a(n) \Lambda_{k}(n)=k H A(x)(\log x)^{k-1}\left\{1+O\left(\delta(\log \delta)^{4}\right)\right\},
$$

avec

$$
H=\prod_{p}(1-g(p))\left(1-\frac{1}{p}\right)^{-1},
$$

la constante $O$ dépendant au plus de $c, k$ et $f$.

Pour démontrer le Corollaire 2, on choisit pour $a(n)$ la fonction caractéristique des entiers $n$ tels que $s_{r}(n)$ soit congru à $b$ modulo $d$. On prend

$$
A(x)=\sum_{n<x, s_{r}(n) \equiv b(\bmod d)} 1, \quad f(d)=g(d)=1 / d .
$$

La fonction $A(x)$ vérifie donc $A(x) \sim x / d$ pour $x \rightarrow \infty$, et le Théorème affirme que l'hypothèse $\left(\mathrm{H}_{1}\right)$ est vraie pour $z=x^{2\left(1-\vartheta_{r}\right)}$, d'où le choix $\delta=$ $2\left(1-\vartheta_{r}\right)=O(\log \log r / \log r)$ grâce à $(6.3)$. On obtient alors directement le Corollaire 2, puisque la contribution à la somme étudiée des entiers divisibles par un carré est en $o\left(x(\log x)^{k-1}\right)$. 


\section{Bibliographie}

[Bo1] E. Bombieri, The asymptotic sieve, Rend. Accad. Naz. Sci. XL Mem. Mat. (5) $1 / 2(1975-76), 243-269$.

[Bo2] -, On twin almost primes, Acta Arith. 28 (1975), 177-193 et Corrigendum, 457-461.

[Fo] E. Fouvry, Sur le problème des diviseurs de Titchmarsh, J. Reine Angew. Math. $357(1985), 51-76$.

[Fo-I] E. Fouvry and H. Iwaniec, Gaussian primes, preprint.

[Fo-M] E. Fouvry et C. Mauduit, Sommes des chiffres et nombres presque premiers, Math. Ann. 305 (1996), 571-599.

[Fo-T] E. Fouvry et G. Tenenbaum, Répartition statistique des entiers sans grand facteur premier dans les progressions arithmétiques, Proc. London Math. Soc. (3) 72 (1996), 481-514.

[Fr-I1] J. Friedlander and H. Iwaniec, On Bombieri's asymptotic sieve, Ann. Scuola Norm. Sup. Pisa Cl. Sci. (4) 4 (1978), 719-756.

[Fr-I2] - - , Bombieri's sieve, dans : Conference on Analytic Number Theory, in honour of H. Halberstam, Urbana 1995, à paraître.

[Ge] A. O. Gelfond, Sur les nombres qui ont des propriétés additives et multiplicatives données, Acta Arith. 13 (1968), 259-265.

[Gra] A. Granville, Integers without large prime factors in arithmetic progressions, I, Acta Math. 170 (1993), 255-273.

[Gre] G. Greaves, A weighted sieve of Brun's type, Acta Arith. 40 (1982), 297-332.

[H-R] H. Halberstam and H. E. Richert, Sieve Methods, Academic Press, 1974.

[Iw] H. Iwaniec, Rosser's sieve-bilinear forms of the remainder term-some applications, dans : Recent Progress in Analytic Number Theory, Vol. 1, H. Halberstam and C. Hooley (eds.), Academic Press, 1981, 203-230.

Mathématique, Bâtiment 425

Université de Paris-Sud

F-91405 Orsay Cedex, France

E-mail: etienne.fouvry@math.u-psud.fr
Laboratoire de Mathématiques Discrètes 163, Avenue de Luminy, Case 930 F-13288 Marseille Cedex 09, France E-mail: mauduit@lmd.univ-mrs.fr 